\title{
THE
}

UNIVERSITY

University of Rhode Island

OF RHODE ISLAND

DigitalCommons@URI

3-1981

\section{Anisotropic Quantum Spin Chains}

\author{
J. Kurmann \\ Gerhard Müller \\ University of Rhode Island, gmuller@uri.edu \\ H. Thomas \\ M. W. Puga \\ H. Beck
}

Follow this and additional works at: https://digitalcommons.uri.edu/phys_facpubs

Terms of Use

All rights reserved under copyright.

\section{Citation/Publisher Attribution}

Kurmann, J., Müller, G., Thomas, H., Puga, M. W., \& Beck, H. (1981). Anisotropic quantum spin chains. J. Appl. Phys., 52, 1968-1970. doi :10.1063/1.329586

Available at: http://dx.doi.org/10.1063/1.329586

This Article is brought to you for free and open access by the Physics at DigitalCommons@URI. It has been accepted for inclusion in Physics Faculty Publications by an authorized administrator of DigitalCommons@URI. For more information, please contact digitalcommons-group@uri.edu. 


\title{
Anisotropic quantum spin chains
}

\author{
J. Kurmann, G. Müller, a) and H. Thomas ${ }^{a)}$ \\ Institute for Theoretical Physics, University of Basel, CH-4056 Basel (Switzerland) \\ M. W. Puga' and H. Beck ${ }^{\text {a) }}$ \\ Institute of Physics, University of Neuchâtel, CH-2000 Neuchâtel (Switzerland)
}

\begin{abstract}
We have studied two models for anisotropic quantum spin chains. (i) XY-chain with a field in the plane: The magnetization of the ferromagnet behaves as $h^{1 / 3}$ for small fields, in agreement with scaling laws. The antiferromagnet shows a critical field at which the ground state is a simple Néel state and which separates power law from exponential decay of spatial correlations. (ii) Anisotropic XY-chain: The dynamic zcomponent spin correlation function can be decomposed into a spin wave and a soliton contribution. The nature of quantum soliton excitations is studied and their form compared to soliton solutions of classical equations of motion.
\end{abstract}

PACS numbers: 75.10.Jm, 75.30.Ds, 75.40.Fa, 75.30.Gw

\section{INTRODUCTION}

In recent years, much work has been done on $s=\frac{1}{2}$ spin chains with a Hamiltonian having at least one rotational axis in spin space $[1,2]$. Finite chain calculations, Fermion representation and Bethe ansatz techniques have been combined in order to obtain information about dynamical correlation functions [2]. On the other hand interesting non-linear excitations contribute to the dynamics of classical fully anisotropic spin chains (e.g. planar systems with a magnetic field in the plane). At low $\mathrm{T}$ and in the continuum approximation, the equation of motion of a polar angle of the classical spin is mapped onto a Sine-Gordon (SG) equation which allows for soliton solutions. Their contribution to dynamical correlation functions at low $T$ can then be calculated [3,4,5]. Experimentally the effect of solitons seems to have been seen in the inelastic neutron cross sections $[6,7]$.

Little work has been done for anisotropic quantum spin chains, specially concerning dynamics. The study of such systems would be of great importance for various reasons. First, it would be interesting to elucidate the relevance of the notion of a "soliton" in a quantum system, using the language of eigenstates and correlation functions instead of searching for special solutions of equations of motion, as in the classical case. Second, there are numerous mappings between $1-d$ quantum and 2-d classical systems [1], such that results for $1-d$ quantum correlation functions also provide insight into the behavior of 2-d classical models.

Except for special cases, Fermion representation techniques are not too useful for anisotropic spin chains, since interacting Fermions with a gap (or a "mass") are not easily treated by a Luttinger approximation. We have studied two special cases of an anisotropic XY-chain :

$$
\begin{aligned}
& H=-J \sum_{n=1}^{N}\left\{(1+\gamma) S_{x}(n) S_{x}(n+1)+(1-\gamma) S_{y}(n) S_{y}(n+1)\right. \\
&\left.+h S_{x}(n)\right\}
\end{aligned}
$$

In section 2 we present some new results on static properties for $\gamma=0$, including the existence of a "critical" field, for which the true quantum ground state of the antiferromagnet $(J<0)$ is a simple Neel state. Section 3 presents exact results for a dynamical correlation function for $h=0$ (which case is solvable in the Fermion representation), shedding some light on the nature of quantum solitons.

\section{ISOTROPIC XY-CHAIN WITH AN IN-PILANE FIELD}

We have studied various properties of (1) for $\gamma=0$ by diagonalizing $H$ for finite chains ( $N \leqslant 10$ ) with periodic boundary conditions. Here are a few interesting results :

(a) $\mathrm{J}>0$ (ferromagnetic exchange) : By extrapolating to the limit $\mathrm{N} \rightarrow \infty$, we found the following behavior for the magnetization : $\sigma_{\mathrm{x}} \alpha \mathrm{h}^{1 / \delta}$ for small $\mathrm{h}$ with $\delta=3$. This is in agreement with the dimensional scaling law

$$
\delta=-1+2 d /(d-2+n)
$$

and $n=3 / 2$, since in the isotropic chain $(h=0)$ correlations decay as [8]

$$
<S_{X}(n) S_{X}(n+R)>\propto R^{-d+2-n}=R^{-1 / 2} .
$$

Saturation is not reached at any finite field, perturbation theory showing that

$$
\sigma_{x s a t}-\sigma_{x} \propto h^{-2}
$$

for large fields.

(b) I $<0$ (antiferromagnetic coupling) : The magnetrzation for $N=8$, which displays steps, is shown in Fig. 1. The last step always occurs at $h=h_{c} \equiv \sqrt{2}$ with mean magnetization $\sigma_{x}\left(h_{c}\right)=\sqrt{2} / 4$. Above this field, the system seems to behave like an XY ferromagnet. Moreover spin correlation functions for large $\mathrm{R}$ seem to have power law decay - as in (3) - for $h<h_{c}$, but an exponential decay for $h>h_{c}$. At $h_{c}$ we find the surprising but rigorous result that, for any $N$, the ground state is a Neel state of the form 


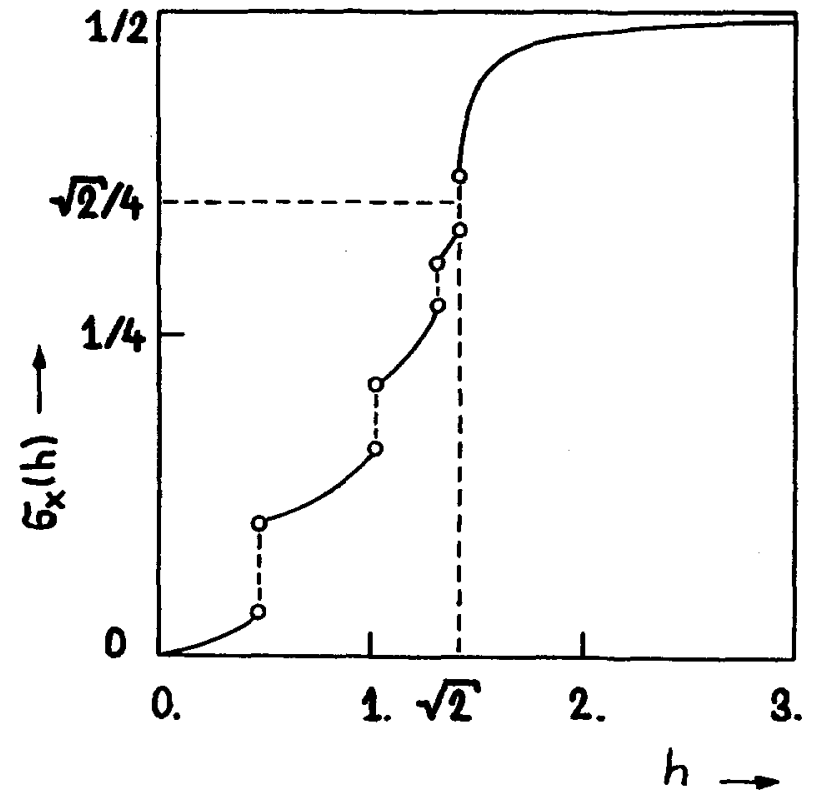

Fig. I Magnetization $\sigma_{x}(h)$ for antiferromagnetic $\mathrm{XY}$-chain of $\mathrm{N}=8$ spins in a field in $x$-direction.

$$
\left|G>=\prod_{n}\right| \psi_{n}>
$$

with

$$
\left|\psi_{n}\right\rangle=\frac{1}{\sqrt{2}}\left[\exp \left(-(-1)^{n} \frac{i \pi}{8}\right)|n \uparrow\rangle+\exp \left((-1)^{n} \frac{i \pi}{8}\right)|n \downarrow\rangle\right]
$$

which is readily verified. Such a "critical" field leading to a "classical" ground state even exists for arbitrary field direction, exchange anisotropies and spin quantum number [9]. The special case of the anisotropic $X Y$ antiferromagnet with a field in z-direction discussed by Barouch and McCoy [10], has two critical fields. At one field the ground state is of the Néel type, at the other the susceptibility diverges. For the isotropic XY model with a field in the plane, these two special fields apparently coincide.

\section{DYNAMICS OF ANISOTROPIC XY-FERROMAGNETS}

Through a Jordan-Wigner and a subsequent Bogolubov transformation Hamiltonian ( 1 ) with $h=0$ can be mapped onto free Fermions with energies [11]

$$
E(k)=J\left[\cos ^{2} k+r^{2} \sin ^{2} k\right]^{1 / 2} .
$$

(The sign of the root is chosen such that $E(k) \rightarrow-J \cos k$ for $\gamma \rightarrow 0$ ). The spin correlation function

$$
G(q, \omega)=\int d t e^{i \omega t} \sum_{n} e^{i q n}\left\langle S_{z}(n t) s_{z}(0,0)>\right.
$$

can be calculated exactly for this model at any temperature $T[12,13]$. Straightforward calculations yield

$$
\begin{aligned}
G(q, \omega)= & \int_{-\pi / 2}^{\pi / 2} d k\left(1+e^{-\beta \varepsilon_{+}}\right)^{-1}\left(1+e^{-\beta \varepsilon_{-}}\right)^{-1} \\
& \cdot\left\{C(k, q) \delta\left(\omega-\varepsilon_{+}-\varepsilon_{-}\right)+e^{-\beta \varepsilon_{+}} D(k, q) \delta\left(\omega+\varepsilon_{+}-\varepsilon_{-}\right)\right\}
\end{aligned}
$$

with

$$
\begin{aligned}
& \varepsilon_{ \pm}=\epsilon(k \pm q / 2) \\
& \varepsilon(k)=+J\left[\sin ^{2} k+r^{2} \cos ^{2} k\right]^{1 / 2}>0 .
\end{aligned}
$$

The functions $C$ and $D$, whose detailed form is not given here for brevity, are determined by the coefficients of the Bogolubov transformation [12]. Equ. (9) is valid for $\omega>0$ and we have

$$
G(q,-\omega)=e^{-\beta \omega} G(q, \omega) .
$$

Corresponding to the two terms in the curly bracket of (9), G contains two contributions, $\mathrm{G}^{(1)}$ and $G^{(2)}$ :

(i) $G(1)_{\text {involves creation of a particle-hole }(p-h)}$ pair with a minimum energy $2 \gamma$. Thus there is a gap in the spectral function: $G(1)=0$ for $\omega<2 \gamma$. On the other hand $G^{(1)} \neq 0$ even at $T=0$, since a $\mathrm{p}$-h pair can be created out of the ground state where all states with negative energy (7) are filled. We call G(1) the "spin wave" or "magnon" part of $\mathrm{G}$.

(ii) $\mathrm{G}(2)$ represents "scattering" of a particle (or hole) from $k+q / 2$ to $k-q / 2$. There is no gap in the spectrum for $q=0$, but $G(2)$ involves $\exp (-\beta E)$ : the particle or hole state is only occupied at finite $T$. We term $G(2)$ "soliton" part of $\mathrm{G}$.

This nomenclature is motivated by interpreting $H$ as the energy of classical spins

$$
\vec{S}=(\sin \theta \cos \phi, \sin \theta \sin \phi, \cos \theta) \text {. }
$$

At low $T$ they will be confined to the $x y$-plane, and for small $\gamma$ the angle $\psi(R, t)=2 \phi(R, t)$ will satisfy, in the continuum approximation, the $S G$-equation $(\mathrm{J}=1)$ :

$$
\frac{\partial^{2} \psi}{\partial R^{2}}-\frac{1}{c^{2}} \frac{\partial^{2} \psi}{\partial t^{2}}=m^{2} \sin \psi
$$

with $c=\sqrt{2}$ and $m=2 \sqrt{\gamma}$. For such classical SG-chains the dynamic correlation functions have been worked out for low $T[3,4]$. There is a "magnon" part (surviving at $T=0$ ), due to small amplitude solutions of (13), and a "soliton" part. The z-component correlations due to solitons can be brought into the form [4] :

$$
\begin{gathered}
G_{c l}(q, \omega) \propto \int d k e^{-\frac{16 \beta}{\sqrt{\gamma}} \mu(k)} \delta(\omega-q \sqrt{2} V(k)) \\
\cdot\left(\frac{V(k)}{\cosh \frac{\pi q \sqrt{\gamma}}{4 \mu(k)}}\right)^{2}
\end{gathered}
$$

with $\beta=\left(k_{B} T\right)^{-1}, \mu(k)=\left(k^{2}+\gamma^{2}\right)^{1 / 2}$,

$$
\mathrm{V}(\mathrm{k})=\frac{d \mu}{d k}=\mathrm{k}\left(\mathrm{k}^{2}+\gamma^{2}\right)^{-1 / 2} \text {. }
$$

The last factor is the square of the "soliton form factor", the q-Fourier transform of the shape of $\frac{\partial \psi}{\partial t}(R)$ corresponding to the soliton solution $\psi(R, t=0)$ of $(13)$ [4].

Evaluating the quantum soliton part, $G(2)$, of (9) for low $\mathrm{T}$ and small $\mathrm{q}$ (using the continuum approximation $E(k) \approx \mu(k))$ yields

$$
\underset{q u}{G(2)}(q, \omega) \propto \int \alpha k e^{-\beta \mu(k)} \delta(\omega-q V(k))\left|\tilde{F}_{q u}(k, q)\right|^{2} .
$$


It obviously has the same structure as the classical result (14). We want to comment and to interpret this fact in the following way :

(i) (14) and (15) still differ in two respects. There are different factors in the exponent : the classical soliton "rest mass" is $8 \mathrm{~m}=16 \sqrt{\gamma}$, whereas in the quantum system it is given by $\gamma$, the minimum energy necessary to create a particle or a hole. The different $\gamma$-dependence of the soliton energy is the effect of quantum mass renormalization [14]. Moreover the velocities occurring in the $\delta$-function differ by $\sqrt{2}$. Here we simply change the scales of length, time and energy in such a way that (14) has precisely the form (15) in order to compare them in a direct way (as we do in Fig. 2).

(ii) The main difference lies in the function $\tilde{F}_{q u}$, which differs from the form factor in (14). We propose to interpret $\tilde{\mathrm{F}}_{\mathrm{qu}}$ as the form factor of a "quantum soliton" and find its real space "shape function" $F_{q u}(R-v(k) t, k)$ by Fourier transformation. Fig. 2 shows a comparison of $F_{c l}(R, k) \propto(\cosh (2 \mu(k) k / \sqrt{\gamma}))^{-1}[4]$ and $F_{q u}(R, k)$ for $\gamma=0.1$ and $k=0.2$. For these parameter values the "quantum soliton" has steeper slopes near $R=0$ (due to a slower decrease of $F_{q u}(k, q)$ for $\left.q \rightarrow \infty\right)$, and is somewhat less localized, having small oscillations in the wings.

(iii) Obviously it is interesting to ask what role the entity "soliton" - which we have identified so far by comparing classical and quantum correlation functions - actually plays in a quantum spin system. It cannot simply be found as a "solitonshaped" eigenstate of $\mathrm{H}$, because the eigenfunctions of (1) are "delocalized" due to translaticnal invariance. However, our dynamic correlation function can be represented as

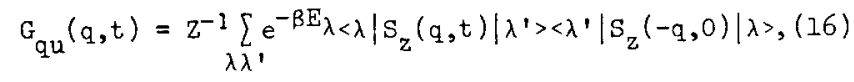

$|\lambda\rangle$ being an eigenstate of $H$ with eigenvalue $E_{\lambda}$. At low T the "soliton part" $G(2)$ is found by choosing, for $|\lambda\rangle$ and $\left|\lambda^{\prime}\right\rangle$, states with a single particle or hole at wavenumber $k$, respectively $k^{\dagger}$, on top of the filled Fermi sea. The relevant matrix elements will then have the form

$$
\begin{aligned}
& \left\langle k\left|S_{z}(q, t)\right| k^{\prime}\right\rangle=\delta_{k-k^{\prime}, q} M\left(\frac{k+k^{\prime}}{2} ; q\right) e^{i\left(\varepsilon(k)-\varepsilon\left(k^{\prime}\right)\right) t} \\
& \text { and, for small } q\left(K \equiv\left(k+k^{\prime}\right) / 2\right): \\
& M(k, q) e^{i\left(\varepsilon(k)-\varepsilon\left(k^{\prime}\right)\right) t} \approx M(K, q) e^{i V(K) q t}
\end{aligned}
$$

Then (16) is written as

$$
\mathrm{G}_{\mathrm{qu}}(\mathrm{qt})=\mathrm{z}^{-1} \sum_{\mathrm{K}} e^{-\beta \mu(K)} e^{i \mathrm{~V}(\mathrm{~K}) \mathrm{qt}}|\mathrm{M}(\mathrm{K}, \mathrm{q})|^{2}
$$

and (as long as we only account for small q) the Fourier transform of $M$ has the form of a travelling wave

$$
\begin{aligned}
& \sum M(K, q) e^{i q(V(K) t-R)}= \\
& \left.\quad=\sum_{\substack{k-k^{\prime} \\
q}}<k\left|S_{z}(q, t)\right| k^{\prime}\right\rangle=F_{q u}(R-v(K) t \mid K)
\end{aligned}
$$

Its shape and velocity are determined by the "center of mass wave number" $K$. According to these arguments, the quantity which bears the typical "solitary wave features" in the quantum spin chain is a matrix element of the time dependent spin operator between certain eigenstates of H.

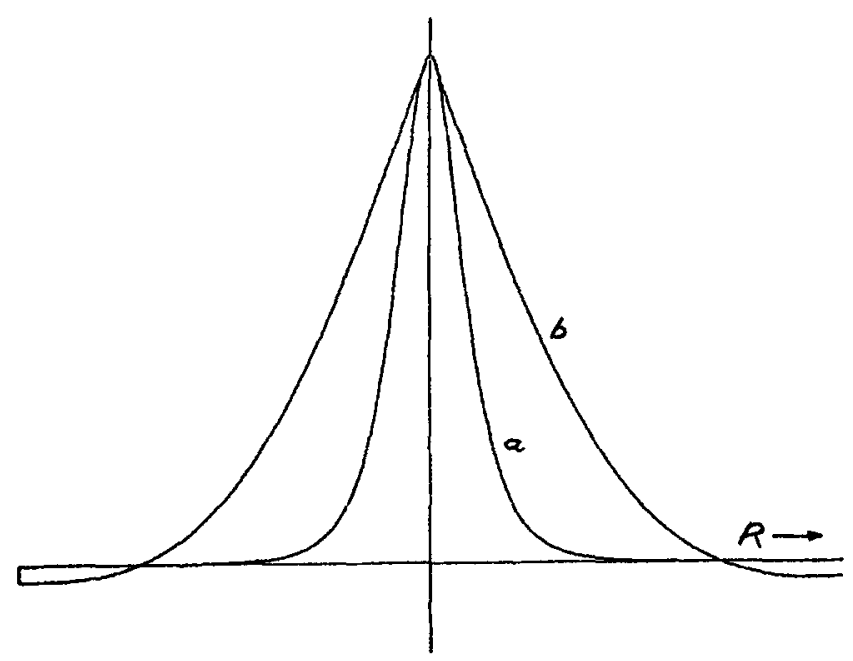

Fig. 2 Comparison of the "shape function" $F(R, k)$ of a classical (a) and a quantum (b) soliton excitation $(\gamma=0.1, k=0.2)$

\section{REFERENCES}

a) This work was supported by the Swiss National Science Foundation.

1. J.C. Bonner, Proc. Int. Conference on "Physics in one dimension", ed. T. Schneider, J. Bernasconi, Springer, 1980 (to be published).

2. H. Beck, M.W. Puga, G. Müller, J. Appl. Physics (this volume).

3. H.J. Mikeska, J. Phys. C 11, L29 (1978).

4. K.M. Leung, D.L. Huber, Sol. State Comm. 32, 127 (1979).

5. H.J. Mikeske, J. Phys. C 13, 2913 (1980).

6. J.M. Kjems, M. Steiner, Phys, Rev. Lett. 느, 1137 (1978)

7. J.P. Boucher, L.P. Regnault, J. Rossat-Mignod, J.P. Renard, J. Bouillot, W.G. Stirling, Sol. State Comm. 33, 171 (1980).

8. B.M. McCoy, Phys. Rev. 173, 531 (1968).

9. J. Kurmann, G. Müller, H. Thomas, (to be published).

10. E. Barouch, B.M. McCoy, Phys. Rev. A 3, 786 (1971)

11. E. Lieb, T. Schultz, D.C. Mattis, Ann. Phys. (N.Y.) 16, 407 (1961).

12. Th. Niemeijer, Physica 36, 377 (1967).

13. S. Katsura, T. Horiguchi, M. Suzuki, Physica 46, $67(1970)$.

14. K. Maki, "Solitons in Low Temperature Physics", to appear in Progr. Low Temp. Phys., vol. VIII. 